\title{
In der Sprechstunde
}

Wie (kritische) Normenforschung ihre Stimme wiederfinden kann

Sozialwissenschaftliche Forschung kann nicht neutral sein. Sie ist, so die Grundhaltung dieses Aufsatzes, immer an der (Re-)Produktion sozialer Wirklichkeit beteiligt und somit als politische Praxis zu verstehen. Aus dieser Perspektive setzt sich der vorliegende Aufsatz mit der konstruktivistischen Normenforschung auseinander. Wir argumentieren, dass konstruktivistische Normenforschung insofern politisch ist, als sie westliche Wertbestände tendenziell reproduziert und ihre globale Hegemonie unhinterfragt lässt und stärkt. Diese politische Haltung wird in der Normenforschung jedoch kaum reflektiert. Demgegenüber ist es unser Ziel im zweiten Teil des Aufsatzes, mögliche Wege aufzuzeigen, wie eine reflexive und kritische Normenforschung betrieben werden könnte. Dafür schlagen wir ein poststrukturalistisch inspiriertes, kritisches Forschungsprogramm vor, das auf drei zentralen forschungspraktischen Schritten aufbaut: erstens der Hinterfragung global hegemonialer Wertbestände, zweitens der Rekonstruktion alternativer Wissensbestände und drittens der expliziten Reflektion der eigenen Forschungsperspektive.

\section{Prolog}

Sofie hat vor einem Monat ihren Abschluss in Politikwissenschaft gemacht und möchte nun promovieren. Sie ist auf dem Weg zu Professor Sapere, Professor für Internationale Beziehungen, der schon ihre Abschlussarbeit betreut hat. Es ist später Nachmittag und Sofie hat den letzten Termin für die Sprechstunde erhalten. Die Tür ist angelehnt und sie klopft. Prof. Sapere blickt auf und bittet sie herein. Sofie gehörte zu seinen besten Master-Studierenden, er würde sie gern auch als Doktorandin weiterbetreuen. Sie nehmen am Besprechungstisch Platz.

Prof. Sapere: Sie haben mir geschrieben, dass Sie sich für eine Promotion interessieren. Was haben Sie sich denn bis jetzt überlegt, Sofie?

Sofie (etwas nervös): Nun, ich interessiere mich für den aktuellen Konflikt in Libyen, genauer gesagt die Tatsache, dass die internationale Gemeinschaft unter UNMandat, der so genannten Responsibility to Protect (R2P), in Libyen eingegriffen hat. (Prof. Sapere nickt interessiert) Die R2P-Norm spiegelt ein gewandeltes Verständnis von staatlicher zu menschlicher Sicherheit wider, in der individuelle Sicherheit höher eingeschätzt wird als staatliche Souveränität. R2P kann jedoch sehr unterschiedliche Auswirkungen auf die individuelle Sicherheit haben. Mich interessiert daher, welche Individuen in welchem Kontext geschützt werden. Am Fall Libyens möchte ich dann der Frage nachgehen, wie libysche Flüchtlinge Unsicherheit 
erleben und wie diese empfundene Unsicherheit in den UN-Sicherheitsdiskurs von R2P passt ... (schaut Prof. Sapere fragend an)

Prof. Sapere: Hm, ich finde den Fall von R2P und den Wandel eines Sicherheitsverständnisses am Fall Libyens sehr interessant, aber wie wollen Sie denn die Analyse individueller Unsicherheit umsetzen?

Sofie: Das habe ich im Detail jetzt noch nicht ausgearbeitet, aber ich dachte vielleicht an teilnehmende Beobachtung und Grounded Theory. Ich habe im Seminar zu feministischen Ansätzen davon gelesen ...

Prof. Sapere (unterbricht etwas ungeduldig): Inwieweit kann denn die empfundene Unsicherheit von libyschen Flüchtlingen neue Erkenntnisse für die Disziplin der Internationalen Beziehungen liefern? Ich verstehe noch nicht recht, wo die Reise hingehen soll: Wollen Sie eine Wirkungsanalyse der Intervention machen? Oder geht es um unbeabsichtigte Effekte? Was ist denn hier genau die abhängige Variable?

Sofie (zögerlich): Ich möchte nichts von dem machen. Ich verstehe das Erlebnis libyscher Flüchtlinge als einen spezifischen Kontext diskursiver Praktiken von Sicherheitspolitik und ich erhoffe mir davon ein besseres Verständnis des Konzepts von Sicherheit, oder vielleicht besser Unsicherheit in den IB, wie es am Beispiel von R2P zu finden ist. Es geht mir um eine Hinterfragung des vorherrschenden IBSicherheitskonzeptes.

Prof. Sapere (überlegt): Sie sagten, dass R2P ein verändertes Sicherheitsverständnis widerspiegelt, nicht wahr? (Sofie nickt.) Das ist für mich ein interessanter und klarer Fall von Normenwandel und ich würde Ihnen vorschlagen, dass Sie sich erst einmal mit der IB-Normenliteratur auseinandersetzen, bevor Sie sich den methodischen Problemen zuwenden. Damit können Sie diesen Fall in die Normenliteratur einordnen und als einen Prozess von Normenwandel im Bereich internationaler Sicherheit konzeptualisieren. (Er geht zum Computer und druckt ein Papier aus.) Hier ist eine Literaturliste zur Normenforschung, die die wichtigsten Titel enthält. Arbeiten Sie die erst einmal durch und dann besprechen wir das weitere Vorgehen in der nächsten Sprechstunde.

\section{Konstruktivistische Normenforschung}

Normen sind ein zentrales Untersuchungsobjekt konstruktivistischer Forschung in den Internationalen Beziehungen (IB). ${ }^{1}$ Das konstruktivistische Projekt begann in den späten 1980er Jahren als Kritik am vorherrschenden rationalistischen For-

1 Wie jeder Text ist auch der vorliegende Beitrag das Ergebnis zahlreicher Interaktionen und Austauschprozesse. Für hilfreiche Anregungen, konstruktive Kritik und viele Hinweise danken wir den Teilnehmenden der Workshopreihe zur Kritischen Normenforschung, und hier insbesondere den organisierenden KollegInnen aus Frankfurt/Main, Duisburg und Hamburg. Desweiteren danken wir dem Redaktionsteam der ZIB sowie den beiden anonymen GutachterInnen. 
schungsprogramm. ${ }^{2}$ Ziel dieser Forschung war es zunächst, empirisch zu zeigen, dass ideelle Faktoren in der internationalen Politik von Bedeutung sind und die Kausalmechanismen und Prozesse herauszuarbeiten, durch die Normen, Kultur und Identität Verhalten beeinflussen (Klotz 1995: 460; Katzenstein 1996: 5; Ulbert 1997: 13).

Normen, die allgemein als »standard of appropriate behaviour for actors with a given identity $\ll^{3}$ verstanden und als zentraler Bestandteil der ideellen internationalen Struktur betrachtet wurden, rückten bald ins Blickfeld vieler konstruktivistischer ForscherInnen. Frühe Studien fokussierten sich primär auf den strukturellen Charakter von Normen und untersuchten ihren Einfluss auf das Verhalten von Akteuren, um zu zeigen, dass »norms matter« (Katzenstein 1996: 30; March/Olsen 1998: 958). Normen, so wurde argumentiert, beeinflussen die internationale Politik in zweierlei Hinsicht: Einerseits sind sie konstitutiv, indem sie die Interessen und Identitäten von Akteuren (insbesondere Staaten) formen (Checkel 1998: 326). So konstituiert etwa die Souveränitätsnorm den Nationalstaat als zentralen Akteur internationaler Politik und versieht ihn damit mit beachtlicher agency (Kowert/Legro 1996: 466). Zum anderen ist sie regulativ und begrenzt den staatlichen Handlungsspielraum, indem sie festlegt, dass nationalstaatliche Grenzen respektiert und legitime (d.h. souveräne) Autoritäten anerkannt werden sollen (Barkin/Cronin 1994: 128).

Mit ihrem Fokus auf Normen als Verhaltensstandards rückte die konstruktivistische Normenforschung als angemessen erachtetes, also an Normen orientiertes Verhalten in den Vordergrund ihrer Untersuchungen. Normgeleitetes Verhalten wurde dabei strategischem, selbstinteressiertem Verhalten von Akteuren gegenübergestellt. Wie James G. March und Johan P. Olsen (1998) argumentieren, handeln Akteure nicht immer nur insofern rational, als sie Kosten minimieren und ihren individuellen Nutzen maximieren möchten, sondern auch gemäß einer Logik der Angemessenheit. Sie übernehmen intersubjektiv geteilte Normen, die mit bestimmten Identitäten und Rollen für Akteure verknüpft sind. Diese Identitäten aktivieren spezifische Vorstellungen von gutem und legitimem Verhalten, die wiederum soziales Handeln leiten und formen (March/Olsen 1998: 959; Risse 2003: 107).

Während die Effekte von Normen wichtiger Bestandteil konstruktivistischer Forschung blieben, begann eine wachsende Gruppe von AutorInnen bald damit, sich intensiver mit strukturellem Wandel zu beschäftigen und die Prozesse zu theoretisieren, durch die Normen entstehen und sich global verbreiten (vgl. etwa Sandholtz/Stiles 2009; Sandholtz 2008). Die Forschung zur Entstehung von Normen ist dabei zumeist akteurszentriert (vgl. Finnemore/Sikkink 2001: 393) und vermutet die Triebkräfte dieses Prozesses in den Anstrengungen verschiedener transnationaler Akteure wie Normunternehmern, advocacy networks oder epistemischen Ge-

2 Vgl. Adler (1997); Finnemore/Sikkink (1998); Checkel (1999); siehe auch Widmaier/Park (2012) für einen aktuellen Überblick über konstruktivistische Normenforschung.

3 Finnemore/Sikkink (1998: 891); vgl. auch Katzenstein (1996: 5) sowie Kowert/Legro (1996: 483); Florini (1996: 380). 
meinschaften (Klotz 1995: 463; Risse/Sikkink 1999: 18; Park 2005: 117; Risse 2006: 263-265; Adler/Haas 1992: 368). Diese Akteure, so wird argumentiert, teilen eine normative Haltung oder eine Wissensbasis und agieren gezielt im internationalen System, um neue Normen zu verbreiten. Die konstruktivistische Normenforschung betont in diesem Zusammenhang vor allem diskursives framing und Überzeugungsprozesse als zentrale Handlungsstrategien, mit denen Normunternehmer die Verbreitung von Normen vorantreiben. ${ }^{4}$

Während die konstruktivistische Normenforschung in den letzten Jahren und Jahrzehnten eine breite Resonanz in der Forschergemeinde der IB erfahren hat und ihr Einfluss auf die bzw. Beitrag zur Disziplin unbestreitbar ist, wurde zugleich wiederholt Kritik an dieser Forschung artikuliert, die einerseits auf analytische Probleme und Versäumnisse hinweist, andererseits aber auch auf die zumeist nicht reflektierte Normativität und Realitätspolitik der Normenforschung aufmerksam macht. Ein Kritikpunkt liegt etwa darin, dass die konstruktivistische Normenforschung von einem statischen Normenkonzept ausgeht. Normen würden hier oft als Dinge (Krook/True 2012: 104) betrachtet, deren Inhalt relativ stabil bliebe und nur verbreitet werden müsse. Dadurch, so die Kritik, blieben aber die generelle Umstrittenheit von Normen und die permanenten Aushandlungsprozesse unsichtbar, durch die normative Bedeutungen produziert und verändert würden. ${ }^{5}$

Weiterhin wurde bemängelt, dass der analytische Fokus der frühen konstruktivistischen Normenforschung auf der bloßen Verbreitung internationaler Normen liege. Normativer Wandel werde dabei zumindest implizit als dichotome Normenübernahme oder -ablehnung konzeptualisiert (Wiener 2009: 179). KritikerInnen argumentieren weiter, dass diese Forschungsperspektive lokale Kontexte in Adaptionsprozessen vernachlässigt und insbesondere Fragen von Macht kaum behandelt werden (MacKenzie/Sesay 2012: 161). Sozialisierungs- (Risse et al. 1999), Diffusions(Holzinger et al. 2007) oder Überzeugungsprozesse (Deitelhoff 2006) erfassen somit nur unzureichend den Austausch, der zwischen internationalen Normen und lokalen Vorstellungen und Praktiken besteht.

Ein weiterer problematischer Punkt der konstruktivistischen Normenforschung liegt darin, dass sie bei der Erklärung normativen Wandels zuweilen ein akteurszentriertes Argument mit einer strukturalistischen Perspektive vermischt und so den Prozess der Normentstehung außer Acht lässt. So wird der Prozess normativen Wandels primär akteurszentriert erklärt, wenn der Fokus auf die gezielten Aktivitäten von Normunternehmern gelegt wird, die eine »neue« Norm propagieren und global verbreiten. Zugleich impliziert dieses Argument aber insofern eine strukturalistische Perspektive, als die neue Norm als Bestandteil einer ideellen Struktur bereits existieren muss, so dass sie zumindest von den Normunternehmern erkannt und für wünschenswert gehalten werden kann (Renner 2013). Mit dieser Argumentation vernachlässigt die Normenforschung die gegenseitige Konstitution von Ak-

4 Vgl. Finnemore/Sikkink (1998: 898); Keck/Sikkink (1998: 17); Payne (2001: 38); Deitelhoff (2006: 75-77).

5 Vgl. Reus-Smit (2001); van Kersbergen/Verbeek (2007); Wiener (2010); Panke/Petersohn (2011). 
teur und Struktur (Epstein 2008: 91) und blendet den Prozess der Normentstehung aus (Wiener 2007: 49-51; MacKenzie/Sesay 2012: 146).

\section{Intermezzo}

(Vier Wochen später. Sofie ist wieder in der Sprechstunde und geht ihre Notizen für das Gespräch durch. Prof. Sapere setzt sich zu ihr an den Besprechungstisch.)

Prof. Sapere: Sind Sie vorwärts gekommen, Sofie? Erinnern Sie mich noch einmal, worum ging es in Ihrem Projekt?

Sofie: Um die Anwendung von R2P beim UN-Einsatz in Libyen. (Prof. Sapere nickt.) Ich habe die von Ihnen vorgeschlagene konstruktivistische Normenliteratur gelesen und habe mir Gedanken darüber gemacht, inwieweit diese für den Fall anwendbar ist.

Prof. Sapere: Ich bin gespannt. (lehnt sich zurück)

Sofie: Der Fall lässt sich nach der Literatur gut als Normenwandel konzeptualisieren. Demnach geht es um die Anerkennung von Individuen als zu schützende Objekte und damit um eine Veränderung des Nichtinterventionsgebots in einen souveränen Staat. Die Norm ist auf UN-Ebene institutionell als Responsibility to Protect verankert. Aus Sicht der konstruktivistischen Normenforschung könnte man dann danach fragen, wie es zu diesem Wandel kam und welchen Einfluss R2P auf politisches Handeln in lokalen Kontexten nimmt.

Prof. Sapere (nickt zufrieden): Hört sich gut an.

Sofie (zögerlich): Ich finde allerdings die Erfahrung des Individuums in dem Fall der R2P-Norm, die sich ja explizit dem Wohl und der Sicherheit einzelner Subjekte verpflichtet fühlt, sehr wichtig. Die Frage nach den lokalen Kontexten geht mir nicht weit genug und ich denke, dass eine Beschreibung der Unsicherheit libyscher Flüchtlinge ein wichtiger Beitrag für die Sicherheitsforschung und auch die IBNormenforschung leisten könnte.

Prof. Sapere: Mein Problem damit ist, dass Sie hier mit einer normativen Sichtweise an den Analysegegenstand herangehen - nämlich, dass sich R2P nachteilig auf individuelle Sicherheit auswirkt. Natürlich kann die Weltgemeinschaft nicht jedem einzelnen Flüchtling individuell helfen, aber deswegen muss die Norm ja nicht grundsätzlich in Frage gestellt werden! Ich finde Ihr normatives Vorgehen und den Untersuchungsfokus auf das Individuum problematisch. Versuchen Sie doch Ihre Untersuchung stärker empirisch-analytisch aufzuziehen, wie Sie es eingangs anhand der Normenliteratur erklärt haben.

Sofie (entrüstet): Aber die meisten Texte, die ich zur Normenliteratur gelesen habe, sind doch auch ziemlich normativ!

Prof. Sapere (setzt sich auf): Wie meinen Sie das?

Sofie (atmet tief ein): Die IB-Normenforschung scheint mir in großen Teilen stark affirmativ gegenüber den moralischen Werten der Normen, die sie untersucht. Zudem wird der Fokus auf die Erforschung westlicher Normen gelegt, was dazu führt, dass andere, nicht-westliche Normen marginalisiert werden. Die Normenfor- 
schung gibt vor, wissenschaftlich objektiv zu sein, aber tatsächlich ist sie nicht nur inhärent normativ, sondern auch politisch!

\section{Konstruktivistische Normenforschung als politische Praxis}

Während die oben skizzierten Kritikpunkte an der konstruktivistischen Normenforschung vorwiegend analytische Probleme adressierten, sind sie noch in einer anderen Hinsicht wichtig, die sich mit dem Begriff der Wirklichkeitspolitik (Zehfuss 2002: Kap. 5) sozialwissenschaftlicher Forschung beschreiben lässt. Speziell ist hiermit die normative Prägung konstruktivistischer Normenforschung gemeint, die implizit in den wissenschaftlichen Texten und Praktiken enthalten ist, aber zumeist nicht reflektiert wird und dazu führt, dass Forschung selbst zur politischen Praxis wird, die zur Legitimierung und Delegitimierung kultureller Ordnungen und Wertbestände beiträgt.

Hiermit betreten wir ein Feld, auf dem in den letzten zwanzig Jahren vielfach feministische ${ }^{6}$ postkoloniale ${ }^{7}$ und poststrukturalistische ${ }^{8}$ ForscherInnen dominante Wissensbestände und hegemoniale Praktiken der Wissensproduktion in den IB als Disziplin kritisiert haben. ${ }^{9}$ Während diese »dissidenten Stimmen « (Ashley/Walker 1990a) in den IB es Ende der 1980er Jahre noch explizit ablehnten, das von Robert O. Keohane (1988: 392) geforderte klar definierte, reflexive Forschungsprogramm vorzulegen, haben »moderate« oder »gemäßigte« KonstruktivistInnen seit den 1990er Jahren genau dieses Projekt betrieben (vgl. zu diesen Labels Doty 2004: 379). In ihrem programmatischen Aufsatz zum Stand der IB-Normenforschung haben Martha Finnemore und Kathryn Sikkink (1998: 894-909) Eckpunkte eines solchen konstruktivistischen Forschungsprogramms vorgelegt (vgl. Engelkamp/Glaab 2011). Nun ist die Normenforschung sicher nicht der einzige Teilbereich der im weiteren Sinne konstruktivistischen IB-Ansätze, in denen zunächst postmoderne Kritikpunkte nach und nach modernisiert worden sind. Jedoch handelt es sich hier um eine Forschung, die für sich explizit in Anspruch nimmt, sowohl reflexiv zu arbeiten als auch wissenschaftliche Erkenntnisse über normativen Wandel zu produzieren. ${ }^{10}$

6 Vgl. Peterson (1990); Tickner (1997); Sylvester (2000); Ackerly/True (2008).

7 Vgl. Inayatullah/Blaney (2004); Agathangelou/Ling (2009); Grovogui (2010).

8 Vgl. Der Derian/Shapiro (1989); Ashley/Walker (1990b); Campbell (1992); Walker (1993); Doty (1996).

9 Diese Diskussion hat auch im deutschen Kontext Vorläufer: So wurden wichtige Aspekte zur Reflexivität in den Sozialwissenschaften bereits im Positivismusstreit in der Soziologie sowie in Interventionen aus der kritischen Theorie in den 1970er Jahren behandelt, vgl. etwa die Beiträge in Gantzel (1975). Zudem ließen sich hier exemplarisch die ZIBBeiträge von Mathias Albert (1994), Thomas Diez (1998), Maja Zehfuss (1998) und Klaus Schlichte und Boris Wilke (2000) als Belege für Arbeiten deutscher IB-ForscherInnen nennen.

10 Zuletzt wurden vermehrt Artikel und Foren in internationalen Fachzeitschriften publiziert, in denen sich poststrukturalistisch und postkolonial inspirierte AutorInnen kritisch mit der konstruktivistischen Normenforschung auseinandergesetzt haben, vgl. z.B. die Beiträge 
Es stellt sich somit nicht nur die Frage, wo in den theoretischen Modellen und Erklärungsangeboten der Normenforschung analytische Probleme liegen, sondern auch, wie die jeweiligen theoretischen und methodischen Entscheidungen und Praktiken bestimmte Wissens- und Wertbestände stärken oder modifizieren und wie sie dadurch dazu beitragen, dass eine bestimmte Lesart von Realität und legitimer Ordnung denkbar und sagbar wird, während andere Ordnungen und Wertsysteme marginalisiert und delegitimiert werden.

Die realpolitischen Konsequenzen solcher diskursiven Normalisierungspraktiken lassen sich immer wieder aufs Neue beobachten, wenn unterschiedliche kulturelle Wissens- und Wertbestände aufeinandertreffen. Beispiele hierfür wären etwa unterschiedliche Verständnisse und Wertungen von good governance in der Entwicklungszusammenarbeit (Gallagher 2012) oder von Friedenssicherung und militärischer Intervention in Ländern des Globalen Südens (vgl. etwa Platenkamp 2007). Die Auswahl oftmals liberaler, westlicher und affirmativer Normen bestimmt in diesen Fällen nicht nur die Forschungspraxis, sondern sie trägt gleichzeitig zu politischen Diskursen und Agenden bei, die teilweise unintendierte und »destructive policy consequences « mit sich bringen, wie im Fall der amerikanischen Invasion im Irak, die durch Ideen der demokratischen Friedensforschung legitimiert wurde (Smith 2011: 153).

Wir argumentieren im Folgenden, dass die konstruktivistische Normenforschung eine implizit eurozentrische Perspektive einnimmt und dabei im Westen weitgehend akzeptierte Normen naturalisiert und stärkt, während nicht-westliche Wertbestände tendenziell marginalisiert und delegitimiert werden (Epstein 2012: 140-141; MacKenzie/Sesay 2012: 157-159). ${ }^{11}$ Es geht uns dabei nicht darum, eine Diskussion etwa über die Universalität von Menschenrechten zu führen und hierin eine kulturrelativistische Position einzunehmen. Vielmehr argumentieren wir, dass die Repräsentation liberaler Werte als globale Normen in der Normenforschung eine Idealisierung impliziert, die es ermöglicht, die dunklere Seite dieser Normen auszublen-

von Krook/True (2012); Adler-Nissen (2014). Die Beiträge von Epstein (2012), Inayatullah/Blaney (2012a), MacKenzie/Sesay (2012) und Widmaier/Park (2012) sind Teil eines von Charlotte Epstein organisierten Forums in International Studies Perspectives, das auf die Working Group Interrogating the Use of Norms in IR zurückgeht, die im Rahmen der ISA-Konferenz 2011 in Montréal stattgefunden hat.

11 Wenn wir an dieser oder an folgenden Stellen von dem Westen bzw. dem Nicht-Westen schreiben, wenn wir lokale und globale Kontexte unterscheiden oder die Beschränktheit eurozentrischer Wissensbestände kritisieren, möchten wir damit keinesfalls implizieren, diese ließen sich im Sinne geografisch lokalisierbarer Entitäten einfach vorfinden. Derartige Dichotomien werden ohne Zweifel durch Texte wie den Vorliegenden reifiziert. Dennoch halten wir hier an diesen Begriffen fest, um die - aus unserer Sicht - problematische Fokussierung der Normenforschung als Teilbereich einer »narrowly Western social science « zu thematisieren (Acharya 2011: 620). In diesem Sinn haben postkoloniale AutorInnen wie Chakrabarty (1992; 2002) und Randeria (2002) kritisiert, dass zumeist spezifisch europäische Entwicklungspfade als universell gültig proklamiert werden, eine Sichtweise, die zugleich die Geschichtlichkeit von Normen ignoriere. Daraus folgt jedoch nicht, dass kritische Forschung nicht auch aus dem oder über den Westen möglich ist (vgl. hierzu auch die unter Abschnitt 6.3 diskutierten Arbeiten von Said und Chowdhry zur wechselseitigen Verschränkung westlicher und nicht-westlicher Geschichte(n)). 
den (vgl. jedoch Geis 2011 und zuletzt Heller et al. 2012), oder, wie es Naeem Inayatullah und David L. Blaney formulieren: liberale Normen versprechen "progress without tragedy« (Inayatullah/Blaney 2012b: 293).

Die eurozentrische Prädisposition der konstruktivistischen Normenforschung zeigt sich zum einen in der Auswahl der empirischen Untersuchungsgegenstände. So werden solche Normen als globale untersucht und damit als solche anerkannt, die zumeist im Westen als gut empfunden und weitgehend akzeptiert sind, z.B. westliche Konzepte von Demokratie, Menschenrechten, Gerechtigkeit (Risse et al. 1999; Finnemore 1996; Deitelhoff 2006). Weder werden ihre historischen Entstehungskontexte wie etwa Kolonialherrschaft oder Sklaverei untersucht, die die normative Autorität westlicher Wertbestände in Zweifel ziehen könnten (Inayatullah/ Blaney 2012a: 168; vgl. auch Bhambra/Shilliam 2009), noch werden tendenziell nicht-westliche Normen als (gute) Normen identifiziert und untersucht (Towns 2010: 24-27). Die untersuchten westlichen Normen bleiben in ihrer Existenz als (gute) Normen stattdessen weitgehend unhinterfragt und ihre Entstehungs- und Wandlungsgeschichte wie auch ihre Umstrittenheit werden ausgeblendet. Indem aber Kontingenz und Geschichtlichkeit dieser Normen nicht thematisiert werden, bleibt ihre Einbettung in hegemoniale westliche Wertvorstellungen unreflektiert und verhindert ihre mögliche In-Frage-Stellung als gute Normen. Auf diese Weise werden normative Bedeutungen mit ihren Wahrheits- und Gültigkeitsansprüchen naturalisiert und damit auch die Wert- und Wissensbestände gestärkt, in die sie eingebettet sind.

Die affirmative Haltung der konstruktivistischen Normenforschung gegenüber einem hegemonialen Wertgefüge und die damit einhergehende Marginalisierung nicht-westlicher Normen spiegelt sich auch im analytischen Fokus der Normenforschung wider, der zumeist auf der bloßen Verbreitung (Diffusion) internationaler Normen liegt und normativen Wandel zumindest implizit als dichotome Normenübernahme oder -ablehnung konzeptualisiert (Zimmermann 2012: 20-24). Dies zeigt sich zum Beispiel in der Ausblendung von Konflikt und Macht im Prozess normativen Wandels, der oft durch Konzepte wie Lernen oder Überzeugung modelliert wird (Neumann/Sending 2010: 7). Auch werden lokale Werte und Artikulationsformen, die sich gegen hegemoniale (westliche) Normen richten, nicht berücksichtigt und marginalisiert. Wie Amitav Acharya (2004: 242) überzeugend dargelegt hat, führt die Darstellung westlich-liberaler Normen als oftmals kosmopolitisch oder universell gültig dazu, dass jeglicher lokaler Widerstand gegen die Übernahme dieser Normen von vornherein als illegitim oder unmoralisch erscheint. Aus der Perspektive der postkolonialen Kritik basieren normative Ordnungen jedoch immer auf den diskrepanten Erfahrungen von Kolonialmächten und Kolonisierten (Grovogui 2010: 239). Die Anerkennung dieser unterschiedlichen historischen Erfahrungen von Kolonialismus und Widerstand lenkt den Blick auf die Frage nach der Ausübung von Macht und Herrschaft in nicht-westlichen Gesellschaften (Chakrabarty 2002: 303) und ermöglicht es so, die Validität von hegemonialen Normen zu hinterfragen. 
Die implizite normative Abwertung und Delegitimierung lokaler Handlungsmacht wird dadurch verstärkt, dass die Verbreitung scheinbar globaler Normen in lokale Kontexte häufig als Prozess der Sozialisierung neuer Staaten und Gesellschaften in globale Normen konzeptualisiert wird (Epstein 2012: 140-143). Das Konzept der Sozialisierung wird dabei tendenziell als ein teleologischer Prozess des Fortschritts verstanden, der zur Besserung des zu Sozialisierenden beitrage. Sozialisierung werde zudem als unidirektional, also vom Globalen zum Lokalen wirkend konzeptualisiert, so dass lokale Akteure automatisch als passive, reagierende Rezipienten der globalen Norm erscheinen. Durch dieses Modell werden lokale Akteure infantilisiert, indem sie als moralisch leere Subjekte konstruiert werden, deren eigene Werthaltungen und Identitäten vor der Normübernahme ausgeblendet und damit als nichtig erklärt werden (Epstein 2012: 140-143). Normativer Wandel werde im Rahmen des Sozialisierungskonzepts somit nur als Wandel hin zum normativ Besseren verstanden. Dadurch werde der Verlust lokaler Identitäten und Werthaltungen durch die beabsichtigte Übernahme globaler Normen übersehen und lokale Artikulationsformen kontingenter Erfahrungen als Widerstand gegen den (moralischen) Fortschritt interpretiert (Epstein 2012: 143).

Während lokales Handeln, das nicht im Namen globaler Normen ausgeführt wird, in der Normenforschung also tendenziell abgewertet wird, werden zugleich die Handlungen von Normunternehmern implizit bestärkt (vgl. Engelkamp/Glaab 2011). Diese werden als zentrale Akteure für die Diffusion westlicher Normen betrachtet. Normunternehmer werden als moralische Subjekte dargestellt, die entweder strategisch, aber geleitet von Empathie, Altruismus und ideellem Pflichtgefühl handeln (Finnemore/Sikkink 1998: 898; Keck/Sikkink 1998: 14), oder sich im Modus verständigungsorientierten Handelns für die globale Verbreitung guter Normen einsetzen (Deitelhoff 2006: 90-97). Die Verbreitung westlicher Normen basiert auf Überzeugung durch gute Argumente, die in rationalen Diskursen ohne Ausnutzung unterschiedlicher Machtpotenziale kommuniziert werden (Deitelhoff 2009). Dabei wird die Verbreitung westlicher Normen durchweg positiv dargestellt, indem Elemente wie Kooperation, gegenseitiges Verstehen und Fortschritt im Prozess der Normdiffusion betont, Zwang oder die Ausübung finanziellen Drucks als mögliche Triebkräfte normativen Wandels aber nicht ausreichend untersucht werden (MacKenzie/Sesay 2012: 147). ${ }^{12}$

Die normative Prädisposition konstruktivistischer Normenforschung mag nicht beabsichtigt sein. Dennoch betreibt diese Forschung eine ganz bestimmte Realitätspolitik, indem sie diejenigen Bedeutungen, die im Westen weitgehend geteilt und als Normen akzeptiert sind, naturalisiert und stärkt, und dadurch auch die Wissensund Wertbestände legitimiert und reproduziert, in die diese Bedeutungen eingebunden sind. Konstruktivistische Normenforschung bietet damit keinen neutralen, objektiven Blick auf die Welt, die sie untersucht (vgl. hierzu schon Cox 1981: 129).

12 Vgl. hierzu auch den Beitrag von Rebecca Adler-Nissen (2014), in dem sie dem klassischen Sozialisierungsargument der konstruktivistischen Normenforschung das Konzept der Stigmatisierung gegenüberstellt. 
Stattdessen ist sie in die Konstruktion von Wirklichkeit eingebunden und trägt durch das Wissen, das sie produziert, zur Reproduktion und Normalisierung hegemonialer (und primär westlicher) Wissensbestände und Machtstrukturen bei (Smith 2004: 510).

\section{Intermezzo}

Prof. Sapere: Also wenn ich Sie richtig verstehe, würden Sie dann bei Forschung zu R2P anhand der gängigen Normenliteratur kritisieren, dass ein westlich-liberal geprägtes normatives Verständnis von individuellen Rechten als universell dargestellt wird?

Sofie: Genau! Zudem führt die Entscheidung, aufgrund der R2P-Norm in bestimmte Konflikte einzugreifen und in andere nicht, gleichzeitig zu einer Aberkennung der Wichtigkeit der individuellen Sicherheit von marginalisierten Individuen wie Frauen, ethnischen Minderheiten oder Flüchtlingen und deren Ausschluss von einem anerkannten und legitimen Sicherheitsdiskurs.

Prof. Sapere: Doch die IB-Normenforschung wird dann immer auch politisch sein. Das ist der Knackpunkt. Wie wollen Sie dieses Dilemma überwinden?

Sofie: Das stimmt. In Seminaren aus anderen Disziplinen wie der Anthropologie, zu Postkolonialismus oder aus der Gender-Forschung habe ich aber gelernt, dass man andere Forschungsfragen stellen kann und dass andere methodologische $\mathrm{Zu}$ gänge gewählt werden können. Dabei geht es ganz zentral darum, Machtbeziehungen und hegemoniale Wissensbestände kritisch zu hinterfragen. Wichtig ist es dabei vor allem, sich die eigenen normativen Vorannahmen, die eigene normative Ausgangsposition klar vor Augen zu führen. Ich finde es zum Beispiel nützlich, dezidiert kritische Strategien in den Forschungsprozess zu integrieren. Beim Lesen der Normenliteratur habe ich etwa bemerkt, dass ich anders lese und die hegemonialen Wissensbestände stärker hinterfrage, wenn ich die Literatur aus anderen Disziplinen mit der IB-Literatur verbinde. Bei meiner eigenen Forschung kann ich mir auch vorstellen, andere Strategien des Schreibens und individuelle Erzählformen einzubinden. Durch Beobachtung des Lebens von Flüchtlingen und narrative Interviews kann ich Mikropraktiken beschreiben, die eine kritische Perspektive auf Sicherheitsdiskurse geben.

\section{Kritische Normenforschung - Skizze eines Forschungsprogramms}

\subsection{Sozialwissenschaft als Wahrheitsproduktion}

Aus unserer poststrukturalistisch orientierten Perspektive, die davon ausgeht, dass alles Soziale diskursiv vermittelt ist, kann Wissenschaft niemals objektiv oder neutral sein. Der politische Charakter sozialwissenschaftlicher Forschung ist unver- 
meidbar, da jegliche Forschung als politische Praxis, also als Teil der Konstruktion von Realität und damit der Legitimierung der einen und Delegitimierung der anderen sozialen Ordnung zu verstehen ist.

Die poststrukturalistische Diskurstheorie kann als radikale und politische Form konstruktivistischen Denkens verstanden werden, die auf einer »anti-essentialistischen Ontologie« und einer »anti-fundamentalistischen Epistemologie« (Torfing 2005: 13) aufbaut. Ontologisch verwirft sie die Annahme, soziale Wirklichkeit könne auf eine stabile und gegebene Essenz zurückgeführt werden und geht stattdessen davon aus, dass soziale Realität durch Diskurse produziert und stabilisiert, also sozial konstruiert wird. Epistemologisch nimmt sie an, dass es keine gegebene und stabile Wahrheit über die Welt geben kann. Vielmehr bedingen hegemoniale Diskurse, was als wahr oder falsch gilt (Torfing 2005: 14). Aus poststrukturalistischer Sicht ist Diskurs die zentrale konstitutive Kategorie sozialer Realität, da es nur in Diskursen und durch Diskurse möglich ist, Bedeutungen temporär zu stabilisieren und die materielle Umwelt bedeutungsvoll werden zu lassen (Epstein 2008: 4-8; Howarth/Stavrakakis 2000: 2-3; Torfing 2005: 21). Diskurse können als relativ stabile Arrangements verstanden werden, als »structured totalit[ies] (Laclau/Mouffe 2001: 115), in denen Bedeutungen und soziale Identitäten in relationalen Bedeutungssystemen angeordnet werden, wodurch soziale Realität erst möglich wird. Diskurse werden durch Artikulationen produziert und reproduziert, die in jeder sozialen Praxis, linguistisch wie nicht-linguistisch, enthalten sind, da jede soziale Handlung bestimmte Bedeutungen bestätigt und reproduziert, während andere hinterfragt und modifiziert werden (Laclau/Mouffe 2001: 105, 113).

Wie unsere alltägliche Sprache und unsere sozialen Praktiken, so ist auch unsere Forschung eingebunden in die und fundamentaler Bestandteil der Konstruktion und Stabilisierung einer ganz bestimmten diskursiv produzierten Wirklichkeit. Wissenschaftliche Wahrheitsproduktion spielt bei der diskursiven Produktion von Wirklichkeit sogar eine herausragende Rolle, da der wissenschaftliche Diskurs in unserer modernen Gesellschaft fundamental ist für die " >political economy< of truth « (Foucault/Gordon 1980: 131), also für die Produktion dessen, was genuin als Wahrheit akzeptiert wird (Litfin 1994: Kap. 2; Aronowitz 1988: vii). Wissenschaftliche Forschung kann daher nicht als neutrale Praktik und objektive Beobachtung einer existierenden Realität betrachtet werden. Stattdessen sind Forschungspraktiken und theoretische Erklärungen selbst Bestandteile der sozialen Konstruktion von Realität und somit politische Eingriffe in die Art und Weise, wie diese produziert und verändert wird.

Ein neutrales Forschungsprogramm kann es demnach nicht geben. Dennoch ist es möglich, ein Forschungsprogramm zu verfolgen, das sich nicht als neutrale Alternative, sondern als politisches und wissenschaftliches Gegenprogramm zum affirmativen Mainstream der Normenforschung versteht und mit dem eine dezidiert kritische Erforschung (normativer) Bedeutungen betrieben werden kann. Weiter oben wurden drei zentrale Kritikpunkte an der Normenforschung formuliert: Erstens reproduziert und stärkt sie durch ihre Forschung hegemoniale, oftmals westliche Wertbestände und Normen und übersieht Macht- und Konfliktpotentiale, die in de- 
ren Herausbildung und Verbreitung involviert sind; zweitens marginalisiert und delegitimiert sie zugleich alternative, zumeist nicht-westliche Wertgefüge und drittens bleibt sie in ihrer normativen Haltung weitgehend unreflektiert.

Das kritische Gegenprogramm, das wir im Folgenden vorschlagen, nimmt eben diese politische Praxis von Normenforschung explizit in den Blick. Unser zentrales Anliegen ist es dabei, Forschungsstrategien zu integrieren, die entgegen der bisherigen konstruktivistischen Normenforschung hegemoniale Wissensbestände und Diskurse denaturalisieren und hinterfragen, so dass deren politischer Charakter sichtbar, die Wissensbestände also re-politisiert werden. Dafür schlagen wir drei forschungspraktische Schritte vor: Erstens die Denaturalisierung hegemonialer Wissens- und Wertbestände und das Aufzeigen von Konflikt und Exklusion im Prozess ihrer Herausbildung durch eine poststrukturalistisch inspirierte Hegemoniekritik, zweitens die Rekonstruktion und Artikulation nicht-hegemonialer Wissensbestände und normativer Perspektiven durch kontextsensible Forschungsstrategien, die vor allem von feministischen und postkolonialen AutorInnen entwickelt und angewendet wurden, und drittens die dezidierte Reflektion der eigenen ForscherInnenperspektive und ihrer normativen Prädispositionen, die etwa in autoethnographischen Studien dargelegt worden ist.

\subsection{Poststrukturalistisch inspirierte Hegemoniekritik als Praxis kritischer Normenforschung}

Die konstruktivistische Normenforschung untersucht vor allem diejenigen Normen, die im Westen weitgehend als Standards guten Verhaltens akzeptiert sind. Diese werden dann primär daraufhin befragt, durch welche Normunternehmer und mit welchen Strategien sie erfolgreich verbreitet werden. Jedoch werden diese scheinbar guten Normen kaum in ihren Geltungsansprüchen hinterfragt und auf ihre möglicherweise dunkleren und ausschließenden Seiten hin untersucht.

Die poststrukturalistische Diskurstheorie stellt mit ihren konzeptionellen Angeboten eine hilfreiche kritische Alternative zu diesem Vorgehen dar, da sie es ermöglicht, den Prozess nachzuvollziehen, durch den sich hegemoniale Diskurse und damit auch Wertordnungen und Normen herausbilden, und zu beleuchten, welche politischen Konflikte und Exklusionen in diese Hegemonialisierung involviert sind. Dabei geht die poststrukturalistische Diskurstheorie davon aus, dass soziale Realität durch hegemoniale Diskurse produziert wird. Diese sind insofern mächtig, als sie eine bestimmte, historisch kontingente Interpretation sozialer Realität vorübergehend fixieren und institutionalisieren (Howarth/Stavrakakis 2000: 4; Torfing 2005: 14-15). Die produktive Macht hegemonialer Diskurse liegt demnach in ihrer Möglichkeit, eine diskursiv produzierte, dabei aber völlig kontingente soziale Realität als objektiv wahr und gegeben erscheinen zu lassen.

Dies ist aber nur möglich, wenn alternative Konstruktionen von sozialer Realität und politischer Ordnung, die ebenfalls einen Geltungsanspruch erheben, zugleich delegitimiert und ausgeschlossen werden. Die Macht eines hegemonialen Diskurses 
birgt also immer auch eine repressive Seite, da sie alternative Konstruktionen unterdrückt und marginalisiert. Diskurse und alle soziale Objektivität, die von ihnen produziert wird, sind somit inhärent politisch; ihre Formierung impliziert die Ausübung von Macht und der Prozess ihrer Sedimentierung ist die Institutionalisierung von Machtbeziehungen (Howarth/Stavrakakis 2000: 4, 9).

Für die kritische Analyse globaler Normen impliziert dies, dass Normen nicht als Bestandteile einer neutralen sozialen Realität mit gegebenen Geltungsansprüchen betrachtet werden können, sondern dass sie immer das Produkt mächtiger, aber kontingenter Diskurse sind, deren Hegemonie auf dem Ausschluss alternativer Wertordnungen und Verhaltensstandards basiert. Diese anderen, unterdrückten und zugunsten des hegemonialen Diskurses delegitimierten Versionen gilt es in einer kritischen Studie aufzudecken, um die Kontingenz des hegemonialen Diskurses genauso wie seine Macht und Politik zu enthüllen. Wie eine solche kritische Untersuchung hegemonialer Normen aussehen könnte, zeigt etwa Judith Renner (2013) am Beispiel der globalen Versöhnungsnorm.

Die Idee der nationalen Versöhnung von Postkonfliktgesellschaften ist spätestens seit dem südafrikanischen Versöhnungsprozess Mitte der 1990er Jahre zur globalen Norm geworden (Moon 2008: 2; vgl. auch Christodoulidis/Veitch 2007: 1; Hirsch 2007: 186). Versöhnung gilt seitdem für Postkonfliktgesellschaften als wünschenswert und durch sogenannte Wahrheits- und Versöhnungskommissionen zu erreichen, in der Opfer und Täter vergangener Menschenrechtsverbrechen öffentlich über ihre jeweiligen Erfahrungen berichten und dadurch von ihren Traumata geheilt und schließlich versöhnt werden (Humphrey 2002: Kap. 8; Moon 2008: 4-5; Renner 2013).

Zahlreiche Akteure setzen sich als Normunternehmer global für die Verbreitung der Versöhnungsnorm ein. Aus Sicht der Normenforschung wäre es daher zunächst nahe liegend, zu untersuchen, mithilfe welcher diskursiven Strategien sie dabei vorgehen und inwiefern sie bei der Normdiffusion erfolgreich sind. So untersucht etwa Michal B.-J. Hirsch (2007: 184-201), inwiefern Transitional Justice-ForscherInnen im Sinne einer epistemic community an der Verbreitung der Versöhnungsnorm beteiligt waren.

Aus poststrukturalistischer Perspektive lässt sich dagegen fragen, wie es überhaupt möglich wurde, dass Versöhnung zu einem anerkannten autoritativen Wert wurde und inwiefern die Privilegierung von Versöhnung auf Kosten anderer Werthaltungen erreicht wurde, die im Laufe der Hegemonialisierung ausgeschlossen und marginalisiert wurden. Wie Renner (2013: Kap. 2) in ihrer genealogischen Analyse des Versöhnungsdiskurses in Südafrika zeigt, weist die Herausbildung dieses Diskurses - und damit die Entstehung der heutigen Versöhnungsnorm - einige Aspekte auf, die einerseits die Geltungsansprüche dieser Versöhnungsnorm in Zweifel ziehen und andererseits ihre repressive Macht enthüllen: So war die Artikulation der heute dominanten Interpretation von Versöhnung im Sinne von öffentlichen Wahrheitsprozessen von diversen Brüchen und Zäsuren geprägt, die zeigen, wie kontingent diese Interpretation und wie hinterfragbar damit ihr Geltungsanspruch ist. Der Begriff Versöhnung ist in den Transitionsverhandlungen Südafrikas über 
längere Zeit mit wechselnden Bedeutungen versehen worden. Unter anderem wurde Versöhnung als die Suche nach politischen Kompromissen interpretiert, als Ende des Apartheid-Regimes, als Anerkennung des politischen Widerstandes gegen dieses Regime sowie auch als Freilassung aller politischen Gefangenen (Renner 2013: Kap. 2). Die Herausbildung unserer heutigen Interpretation von Versöhnung als Prozess der Vergebung und Heilung durch öffentliches Wahrheit-Sprechen wurde erst später, nach der Verabschiedung der Übergangsverfassung, artikuliert. Versöhnung im Sinne einer öffentlichen Aufarbeitung der Vergangenheit ist also weder feststehend noch notwendig und konnte sich dennoch als dominante Bedeutung von Versöhnung festigen und global verbreiten.

Zum anderen zeigt die genealogische Analyse, dass die Hegemonie der Versöhnungsnorm als Standard angemessenen Umgangs mit Gewalt und Unterdrückung nur möglich wurde, indem alternative Aufarbeitungsformen ausgeschlossen und delegimiert wurden. So war die Praxis der nationalen Versöhnung zunächst höchst umstritten. Forderungen nach nationaler Versöhnung konkurrierten mit Rufen nach bestrafender Gerechtigkeit, wie sie etwa von den Theologen und Verfassern des Kairos-Dokuments 1985 geäußert wurden. Was Südafrika vor jeder Versöhnung brauche, so die Theologen, sei Gerechtigkeit und die Bestrafung derer, die am unterdrückerischen Regime mitgewirkt haben (Kairos 2007: 50-56; Moon 2008: 3235). Beides, Versöhnung und Bestrafung, waren zunächst gleichermaßen mögliche Wege, um mit der vergangenen Gewalt und Unterdrückung der Apartheid-Zeit umzugehen. Obwohl die Kairos-Theologen nicht allein waren mit ihrer Forderung nach Gerechtigkeit und Bestrafung, wurden solche Forderungen mit der Privilegierung des Versöhnungsdiskurses marginalisiert.

Den Forderungen nach bestrafender Gerechtigkeit wurde die Idee nationaler Versöhnung gegenübergestellt, etwa von der Nationalpartei von Präsident Frederik Willem de Klerk, die Versöhnung als einzige Möglichkeit für ein geeintes Südafrika propagierte (de Klerk 2007: 66). Die Hegemonialisierung dieser Versöhnungsforderungen gegenüber den Rufen nach bestrafender Gerechtigkeit wurde nur möglich, indem die Forderungen nach Gerechtigkeit Schritt für Schritt aus dem Spektrum legitimer Handlungen ausgeschlossen und Bestrafung als Rache und Fortsetzung der Gewalt interpretiert wurden. Dies führte zur Delegitimierung von Bestrafung und machte Versöhnung durch Amnestie und einen öffentlichen Wahrheitsprozess zur einzig legitimen Form der Vergangenheitsbewältigung in Südafrika.

Im Zuge der globalen Diffusion der Versöhnungsnorm werden nun alternative Umgangsformen mit der Vergangenheit auch in anderen Post-Konflikt-Gesellschaften ausgeschlossen. Am Beispiel Sierra Leone zeigt Renner (2013: Kap. 5), wie Forderungen der lokalen Bevölkerung, die Vergangenheit ohne Aufarbeitung hinter sich zu lassen, durch den Versöhnungsdiskurs marginalisiert wurden, da öffentliche Wahrheitsprozesse als legitime und scheinbar richtige Art der Vergangenheitsaufarbeitung gelten. Lokalen Bevölkerungsgruppen, die sich der Versöhnungspraxis dennoch verweigern wollten, blieb damit nur noch der kollektive Boykott der öffentlichen Anhörungen, da ihre Forderungen nach einem alternativen Umgang mit 
der Vergangenheit vom Versöhnungsimperativ unterdrückt wurden (vgl. Renner 2013: Kap. 5; MacKenzie/Sesay 2012: 15; Shaw 2005: 8-9).

Zusammenfassend bietet die poststrukturalistische Perspektive gegenüber der konstruktivistischen Normenforschung einerseits einen Weg, scheinbar akzeptierte Normen zu hinterfragen und als Bestandteil historisch kontingenter Diskurse zu begreifen. Andererseits hilft sie dabei, die dunklere Seite hegemonialer Normen zu beleuchten und zu zeigen, dass diese Hegemonie nur auf Kosten alternativer Handlungsweisen und Verhaltensstandards möglich ist, die aus dem Spektrum legitimer Handlungen ausgeschlossen werden. Die poststrukturalistische Kritik hegemonialer Wertbestände ist ein erster möglicher Schritt eines kritischen Normenforschungsprogramms. Die erwähnten marginalisierten Handlungsweisen und Forderungen bewusst in die Analyse zu integrieren, ist dann der zweite Schritt, den wir im folgenden Abschnitt vorschlagen.

\subsection{Rekonstruktion alternativer Wissensbestände durch feministische und postkoloniale Forschungspraktiken}

Neben der Denaturalisierung globaler hegemonialer Wertbestände schlagen wir als zweiten Schritt unseres kritischen Normenforschungsprogramms vor, die in der konstruktivistischen Forschung marginalisierten lokalen Wertbestände zu rekonstruieren und zu stärken. Hierzu kann eine kritische Normenforschung etwa auf die Strategien von postkolonialen und vor allem feministischen IB-AutorInnen zurückgreifen, die die Reflexivität und Normativität ihrer eigenen Forschung vermutlich am klarsten herausgearbeitet und theoretisiert haben. Die Aufgabe, hegemoniales Wissen zu hinterfragen, ist dabei eingebettet in eine explizit formulierte feministische research ethic (vgl. Ackerly et al. 2006: 225; Ackerly/True 2008: 693). In ihren Arbeiten haben diese AutorInnen den Forschungsfokus auf die konkreten Praktiken eines Diskurses verschoben und Formen des kritischen Lesens oder des wirklichen Zuhörens (Park-Kang 2011) als kritische Techniken etabliert, um marginalisierte Positionen und Perspektiven zu untersuchen.

Unser oben formulierter Vorschlag, kritische Normenforschung als poststrukturalistische Hegemoniekritik zu betreiben, erfordert daher zunächst, eine andere Form des Lesens als kritische Strategie zu praktizieren. Der postkoloniale Theoretiker Edward Said schlägt hierzu eine »kontrapunktische Lektüre« des westlichen kulturellen Archivs vor, welches unter dem »Bewußtsein [sic] der Gleichzeitigkeit der metropolitanischen Geschichte, die erzählt wird, und jener anderen Geschichten, gegen die (und im Verein mit denen) der Herrschaftsdiskurs agiert« (Said 1994: 92) passieren müsse. Inspiriert von der klassischen Musik bedeutet der Kontrapunkt in Saids Metapher das organisierte Wechselspiel zeitweilig privilegierter Themen, die zusammen aber eine ganze melodische Ordnung bilden. Diese Art des Lesens beinhaltet somit eine Berücksichtigung der Herrschaftspraktiken als auch des Widerstands, der im westlichen kulturellen Archiv herausgeschrieben wurde (Chowdhry 2007: 102-106; Glaab/Engelkamp 2012). Für den Kontext einer kritischen Normen- 
forschung bedeutet dies, nicht einfach bestimmte Wertvorstellungen als allgemein gültige Standards vorauszusetzen, an dem lokale Kontexte verglichen und bewertet werden. Vielmehr gilt es, unterschiedliche Wissensbestände in ihrer wechselseitigen Konstitution und Kontingenz herauszuarbeiten.

Als Beispiel für eine solche Forschung kann die Arbeit von Siba Grovogui (2011) zur Bedeutung von Menschenrechten dienen. In der konstruktivistischen Normenforschung werden Menschenrechte zumeist als Normen mit universellem Geltungsanspruch konzeptualisiert. Kodifiziert als internationale Rechtsnormen gelten insbesondere liberale Freiheitsrechte wie das Wahlrecht, das Recht auf freie Meinungsäußerung oder das Folterverbot. Forschungspraktisch gelangt dabei die Diffusion dieser Menschenrechtsnormen durch internationale Organisationen und transnationale Netzwerke in nationalstaatliche Kontexte in den Blick (Forschungsgruppe Menschenrechte 1998; Risse et al. 1999; Hafner-Burton/Tsutsui 2005). Durch Sozialisierungsprozesse soll der normverletzende Staat zu einer Institutionalisierung und Internalisierung internationaler Menschenrechte gelangen, die einen Wandel zu einer besseren Menschenrechtspraxis bewirken (vgl. etwa das Spiralmodell der Forschungsgruppe Menschenrechte 1998; Risse et al. 1999). Varianz bei der Implementierung von Menschenrechtsnormen wird laut dieser Forschung nicht auf divergierende Wertbestände zurückgeführt, sondern mit einem Verweis auf den unterschiedlichen Stand von Staaten in der graduellen Umsetzung der Norm erklärt.

Grovoguis Arbeit ermöglicht es, diese Art der Forschung zu hinterfragen, indem er die Bedeutung von Menschenrechten für haitische Sklaven im 18. Jahrhundert mit den Konzepten der Menschenrechte kontrastiert, die zeitgleich in Frankreich und in den Vereinigten Staaten als Teil des liberalen Konstitutionalismus etabliert wurden. Demnach entwickelten sich drei unterschiedliche Verständnisse von politischer Subjektivität: während in Frankreich Bürger und in den Vereinigten Staaten Individuen als zentrale Kategorien für Personen und ihre zu sichernden Rechte ausgewiesen wurden, entwickelte sich in Haiti das Modell des Menschen und damit ein umfassenderes Verständnis von Freiheit, das auch für die existentiellen Grundbedürfnisse aller Ausgestoßenen Sorge zu tragen hatte (Grovogui 2011: 48). Die SklavInnen und anderen konstitutionell vormals Ausgeschlossenen, die die haitische Revolution initiierten, sahen somit die Verantwortung des Staates für seine BürgerInnen als eine nicht diskriminierende Sicherung des sozio-ökonomischen Rechts auf Leben. Auf Ebene der Vereinten Nationen (VN/UN) werden heute jedoch bürgerliche und politische Rechte priorisiert und als essentielle Menschenrechte deklariert, während sozio-ökonomische Rechte nur sekundär sind (Grovogui 2011: 63). Grovoguis kontrapunktische Lektüre weist auf die multiplen Genealogien der Menschenrechte hin, westliche Normen seien gerade nicht einzigartig und unumgänglich (Grovogui 2011: 62).

Die Möglichkeit, dass Gesellschaften jenseits des Westens unterschiedliche, aber gleichermaßen valide Vorstellungen von Menschenrechten hervorbringen können, wird aus der universellen Annahme von Menschenrechten, die auf der westlichen Tradition beruhten, herausgeschrieben (Grovogui 2011: 43). Hieraus folgt jedoch keine Relativierung oder Partikularisierung der Idee der Menschenrechte an sich, 
vielmehr sei es $»[\ldots]$ possible as often happens in the global South, to imagine protected human rights as existing outside of Western norms, without neglecting the possibility of universalism or universality, which is the appeal of the concept of human rights « (Grovogui 2011: 62). Grovoguis Perspektive richtet so den Blick der Forschenden auf die Kontingenz scheinbar universeller globaler Normen und ermöglicht damit eine kritische Betrachtung der normativen Prädisposition der eigenen Forschung.

Zur Rekonstruktion marginalisierter Wissensbestände nutzen feministische und postkoloniale Ansätze neben dem kritischen Lesen ethnologische Forschungsmethoden, die sich sensibel gegenüber den SprecherInnenpositionen zeigen. So hat Carol Cohn $(1987 ;$ 2006) in ihren wegweisenden Arbeiten zur sexualisierten Sprache in nationalen Sicherheitsdiskursen ethnographische Methoden angewandt, die auf offenes Zuhören, Miterleben und Reflektion der eigenen Positionierung in Relation zum Untersuchungsgegenstand beruhen. Wirkliches Zuhören gestattet somit das zu hören, was oftmals still und unausgesprochen bleibt (vgl. Kronsell 2006: 110-111). In Cohns Fallbeispiel war dies die diskursive Normalität eines zunächst vollends von seinem Gegenstandsbereich abstrahierten und hochtechnisierten Fachdiskurses über nukleare Zerstörung, der den von Cohn untersuchten Verteidigungsexperten eine scheinbar unhinterfragbare Rationalität zuschrieb. Dieser Diskurs ermöglichte es seinen Sprechern, leidenschaftslos über nuklearen Tod zu sprechen. Während Cohn in ihrem Text ironisch den phallozentrischen Subtext dieses hypermaskulinisierten (Nandy 1988: 35) Spezialdiskurses aufzeigt, liegt die verblüffende Erkenntnis ihrer Arbeit vor allem darin, dass die suggestive Normalität dieser »technostrategischen « Sprache (Cohn 1987: 690) nach einiger Zeit begann, auch ihre Wahrnehmung des Analysegegenstandes zu beeinflussen. Cohn beschreibt, wie sie im gleichen Umfang, in dem sie lernt, die Sprache der Verteidigungsexperten zu sprechen, ein Gefühl von Kontrolle über ihren Untersuchungsgegenstand erfährt (Cohn 1987: 704). Doch ist dieser Diskurs nicht unschuldig, in Cohns Worten: »Technostrategic language can be used only to articulate the perspective of the users of nuclear weapons, not that of the victims « (Cohn 1987: 706).

Im Sinne dieser Forschung ließe sich am oben skizzierten Beispiel der IB-Normenforschung zu Menschenrechten kritisieren, dass in den Diffusions- und Sozialisierungsmodellen die Perspektive der von Menschenrechtsverletzungen betroffenen Personen nicht auftaucht. So weist Spike Peterson darauf hin, dass die liberale, westliche und individualistische Tradition der Menschenrechtsnorm nur direkte Gewalt und nicht die strukturelle Gewalt erfasst, die etwa Frauen durch häusliche Gewalt, Prostitution, Genitalverstümmelung oder Mitgiftmorde erfahren (Peterson 1990: 305). Auch wenn sich an diesen Aspekten wieder andere Festlegungen und Auslassungen identifizieren ließen, bleibt der Kritikpunkt, dass subjektive Unrechtserfahrungen in der modellhaften Sprache von Spiralen, Stadien oder Lebenszyklen abstrahiert werden. Eine ethnologische Strategie, die nicht nur die Perspektive der sozialisierenden Akteure erfasst, vermag somit den Blick auf die von der hegemonialen Deutung der Menschenrechtsnorm Ausgeschlossenen zu richten und ei- 
ne emanzipatorische, kontextsensible und weniger essentialistische Forschung zu ermöglichen.

Die Praxis kritischen Lesens und Zuhörens, die die Erfahrungen unterschiedlicher Subjektivitäten berücksichtigt, lenkt den Blick auf die Kontingenz normativer Ordnungen. So bringt eine Strategie des kritischen Lesens von sich überschneidenden und diskrepanten Erfahrungen neue Deutungen von Normen und Normativität hervor, die nicht unmittelbar an hegemonialen Wertmaßstäben gemessen werden. Kontrapunktisches Lesen stellt in erster Linie andere Fragen an die IB-Normenforschung: Es fragt nach der Herkunft scheinbar globaler Normen, nach den Bedingungen ihrer Durchsetzung und danach, wem und welchem Zweck sie dienen (Grovogui 2010: 234). Die hier illustrierte Strategie des kritischen Lesens geht Hand in Hand mit einem vorsichtigen oder wirklichen Zuhören, das, so Sungju Park-Kang (2011: 875), am Anfang jeder Kritik stehe. Demnach bedeutet wirkliches Zuhören, sich mit dem nötigen Respekt und genügend Zeit auf die Dialogpartnerin zu konzentrieren und den eigenen Kontext zu reflektieren sowie die eigene Selbstpositionierung zurückzunehmen (Park-Kang 2011: 875). Diese Art des kritischen Lesens und Zuhörens ermöglicht ein nicht-essentialistisches und emphatisches Verständnis sozialen Wandels und lokaler Kontingenz, die der Wirklichkeitspolitik von Normenforschung oftmals verborgen bleibt (Grovogui 2010: 244).

\subsection{Reflektion der normativen Prädispositionen von Forschenden durch Autoethnographie}

Die Reflektion der eigenen Perspektive ist der dritte Aspekt, den ein kritisches Normenforschungsprogramm berücksichtigen sollte. Normenforschung ist wie jede Forschung immer Bestandteil einer bestimmten politischen Ökonomie des Wissens. So illustriert etwa die Arbeit von Carol Cohn (1987; 2006) nicht nur die suggestive Macht eines scheinbar rationalen Spezialdiskurses, das Beispiel verweist auch auf ein weiteres zentrales epistemologisches Problem, das aus unserem poststrukturalistischen Rahmen folgt, nämlich auf die Frage der Reflexivität der Forschenden, die im Zuge ihrer Forschung den untersuchten Akteuren bestimmte Subjektpositionen zuschreiben. So werden in unserem Beispiel der südafrikanischen Versöhnungsnorm Praktiken in der Forschung unhinterfragt als gute Praktiken repräsentiert und damit alternative Umgangsformen mit der Vergangenheit unterdrückt. Epistemologische Gewalt wird hier in höchst subtiler Weise ausgeübt, nämlich über eine scheinbar unpolitische Versöhnungsnorm, über die bestimmte Praktiken und Subjektpositionen in die ProtagonistInnen eingeschrieben werden. Diese Praxis als eine Form von Gewaltausübung überhaupt zu erkennen, ist jedoch nicht selbstverständlich. Im Gegenteil erfordert es eine In-Frage-Stellung der eigenen Position als ProduzentIn von Wahrheit, also eine kritische Praxis, die zumindest in den Endprodukten sozialwissenschaftlicher Forschung bislang kaum Berücksichtigung findet (Brigg/Bleiker 2010: 781; Doty 2010: 1048; Inayatullah 2011b: 5-7). Eine Strategie aus der Anthropologie, mit deren Hilfe NormenforscherInnen ihre eigenen Erfah- 
rungen und Perspektiven theoretisch reflektieren können, ist Autoethnographie (Inayatullah 2011a).

»Autoethnography can be defined as a self-narrative that critiques the situatedness of self with others in social contexts « (Spry 2001: 710). Als Technik akademischen Schreibens zielt diese Methode dabei zugleich auf die Rekonstruktion und eine Kritik der eigenen SprecherIn-Position (Brigg/Bleiker 2010: 781). Ein erster Schritt ist es demnach, das sprechende Selbst im Prozess des wissenschaftlichen Schreibens zu aktivieren. Roland Bleiker und Morgan Brigg (2010: 777) kritisieren die Konvention in den IB, die Person des/r Schreibenden als Subjekt, das bestimmte Entscheidungen trifft und somit in vielerlei Hinsicht in den Forschungsprozess involviert ist, aus dem eigentlichen Text herauszuschreiben (vgl. auch Doty 2004; Dauphinee 2010; Löwenheim 2010). Dagegen argumentieren sie für eine explizite Thematisierung der eigenen Situiertheit als Forschende/r. Hierzu sind unterschiedliche Formen denkbar: die Untersuchung des eigenen Falls kann zeigen, wie internationale Politik auf das Individuum in ihrem alltäglichen Leben einwirkt (vgl. Hülsse 2011: 56; Löwenheim 2010: 1023-1025). Die Rekonstruktion der eigenen Lebensgeschichte kann dabei helfen, die Komplexität kontingenter Lebensumstände zu illustrieren und so unterschiedliche »form[s] of being-in-the-world « (Jackson 2011: 169; vgl. auch Kumarakulasingam 2011: 32-37) aufzeigen. So lässt sich etwa die Auswahl einer bestimmten Forschungsperspektive oder Thematik begründen (vgl. Inayatullah 2011b; Chan 2011; Edkins 2011). Ein solches Explizitmachen der eigenen Situiertheit kann aber auch genutzt werden, um einen hegemonialen Diskurs zu dekonstruieren (El Alaoui 2011: 41-44).

Elizabeth Dauphinee (2010) konfrontiert sich (und uns als Lesende) vermutlich am radikalsten mit den ethischen Fragen, die mit einer Problematisierung ihrer Rolle als Wissenschaftlerin einhergehen. In ihrer autoethnographischen Reflektion über ihre Forschung zum Bosnienkrieg hinterfragt sie ihre Verantwortung als Produzentin von Wahrheit, die auf den Lebenserfahrungen ihrer Interviewpartner ihre eigene berufliche Karriere aufbaut. Dauphinee bricht die Frage, wie ihre Forschung den von ihr Erforschten nützt, auf drei aufeinander bezogene Teilaspekte herunter, die sie in Bezug zu dem von Roxanne Doty formulierten Problem der akademischen Stimme (Doty 2004: 378-383; 2010: 1049-1050) setzt: Diese könne erstens soziale Wirklichkeit nur begrenzt wiedergeben; zweitens beinhalte die Repräsentation dieser sozialen Wirklichkeit zu einem gewissen Grad immer die Ausübung epistemologischer Gewalt; und drittens seien es disziplinäre narrative Konventionen, die letztlich bestimmen, was sagbar ist und was nicht (Dauphinee 2010: 805-808).

Autoethnographie lässt sich somit als eine kritische Reflektion über die Wirkungsweise akademischer Konventionen anwenden, zugleich aber auch als In-Frage-Stellen dieser Mechanismen, «a counter technology and mentality, which deconstructs the processes through which one became a governable person « (Löwenheim 2010: 1028). Regierbar meint hier eine Unterwerfung des/r Forschenden unter eine disziplinäre Konvention, nach der die Position des schreibenden Subjekts möglichst durch Stil und Form akademischen Schreibens aus dem Text herausge- 
strichen wird, obwohl eine strikte Trennung von wissensproduzierendem Subjekt und beschriebenem Objekt letztlich unmöglich ist. Naeem Inayatullah nennt dieses Spiel mit der gleichzeitigen Ab- und Anwesenheit des Autors eine prekäre Fiktion, die auf einer fiktiven Distanz der Schreibenden in der akademischen Prosa beruhe. Die Ideen der Schreibenden sprächen hier quasi für sich ohne den Autor oder die Autorin als solche/n zu enthüllen (Inayatullah 2011b: 5). In der Normenforschung wird (wie auch in anderen politikwissenschaftlichen Forschungsfeldern) der biographische Kontext oder die Entstehungsgeschichte eines Textes kaum explizit gemacht (vgl. jedoch Kruzel/Rosenau 1989). Dies wäre jedoch gerade in solchen Fällen, wo WissenschaftlerInnen selbst politisch aktiv oder beratend tätig sind, wünschenswert. ${ }^{13}$

Die Frage, welche Rolle das Selbst in einem wissenschaftlichen Text spielen soll oder darf, wird von AutoethnographInnen durchaus kontrovers diskutiert. Dauphinee etwa warnt vor einem Wettbewerb unter IB-ForscherInnen, "who writes the prettiest sentence about a war zone« (Dauphinee 2010: 817). Brigg und Bleiker betonen dagegen, dass es hier nicht um eine Art politikwissenschaftliches l'art pour l'art geht, sondern um eine theoriegeleitete Auseinandersetzung mit der eigenen Weltsicht und Subjektivität. Dennoch wird eine andere Form des akademischen Schreibens in dieser Forschung oftmals auch als bewusstes Stilmittel benutzt. Feministisch-postkoloniale AutorInnen etwa »performen« in ihren Texten geradezu die Kritik, die sie artikulieren möchten. Ein Beispiel hierfür sind die Texte von Anna Agathangelou und L.H.M. Ling, die in ihren Aufsätzen oft ungewöhnliche Wege gehen, um etwa die Artikulation marginalisierter Stimmen zu unterstützen (Agathangelou/Ling 2004a; 2004b; 2005). Poesie, fiktionale Dialoge und Rollenspiele werden hier als rhetorische Mittel benutzt, um Emotionen zu rekonstruieren, erlebbar zu machen und so das Argument des Textes auch in seiner Form zu unterstreichen. Diese Texte sind zugleich kritische Interventionen, in denen AutorInnen oftmals (auch) einen politischen Standpunkt gegen einen als hegemonial verstandenen Diskurs einnehmen und vertreten.

Wir zögern an dieser Stelle, autoethnographisches Schreiben per se als eine Methode für mehr Reflexivität in der Normenforschung zu betrachten. Dieser Punkt ist auch in der Literatur umstritten: während Brigg und Bleiker (2010) autoethnographisches Schreiben als eine Art reflexive Kontrollmöglichkeit für Wissensproduktion zu betrachten scheinen, sind sich Dauphinee (2010: 813) und Doty (2010: 1049-1050) einig, dass Autoethnographie keine Methode sein könne, mit der sich das Problem der Subjektivität oder der Normativität der Forschenden einfach lösen ließe. Jedoch kann die Perspektive des I in IR (International Relations) zu jeweils individuellen und teils sehr persönlichen Einsichten zum Aufeinandertreffen zwischen Theorie und Gegenstand der I/internationalen Beziehungen führen (Inayatullah 2011b: 9-11).

13 Vgl. zur Forschung über den Demokratischen Frieden und seine politischen Implikationen die Beiträge von Smith (2011) und Geis (2011). 
Demnach ließen sich grob drei Bereiche identifizieren, in denen autoethnographische Strategien einen Mehrwert für kritische Normenforschung versprechen: Erstens wird die Beziehung zwischen dem schreibendem Subjekt, dem Forschungsgegenstand und dem/r LeserIn offen thematisiert und somit der Kritik zugänglich gemacht. Dies kann die Wahl des Forschungsgegenstands selbst betreffen, die ja immer auch die Nicht-Berücksichtigung anderer möglicher Forschungsthemen mit sich bringt (Smith 2004), aber auch eine explizitere Hinterfragung der eigenen epistemologischen Position anleiten. Dies führt zweitens zu einer Re-Politisierung des Forschungsprozesses, etwa wenn die normativen Implikationen der eigenen Forschung wie bei Dauphinee (2010) oder Doty $(2006 ; 2010)$ reflektiert werden. Doty (2010: 1048) plädiert in diesem Zusammenhang dafür, im Prozess des akademischen Schreibens »human connections« zu etablieren, die die Möglichkeit einer formalen Gegen-Erzählung zu hegemonialen IB-Konventionen bieten. Und drittens können ForscherInnen ihre individuellen Erfahrungen als aktivierende und kritische Methoden in der Lehre stärker einbringen. So berichtet Oded Löwenheim (2010: 1044-1045), dass die explizite Thematisierung seiner individuellen Erfahrungen als israelischer Soldat seinen Studierenden tiefere Einblicke in die ethischen Implikationen von Sicherheit in der Lehre ermöglicht habe (vgl. Löwenheim 2010; Stump 2011; Amy 2011).

Um es klar zu formulieren: Natürlich fordern wir an dieser Stelle nicht, dass von nun an NormenforscherInnen nur noch ihre eigenen Erfahrungen zum Gegenstand ihrer Forschung machen sollen. Auch glauben wir nicht, dass eine Hinwendung zu feministischen oder ethnologischen Methoden allein ein Patentrezept für kritische Normenforschung darstellt. Wir denken jedoch, dass unser Vorschlag, kritische Normenforschung als Dekonstruktion hegemonialer Wissensbestände zu betreiben, nicht ohne Auswirkungen auf die Art und Weise sein kann, wie eine solche kritische Wissensproduktion praktiziert wird. Hierzu benötigen wir nicht nur Strategien, um lokales und oftmals marginalisiertes Wissen zu rekonstruieren, wir müssen uns auch der disziplinierenden Auswirkungen bewusst werden, die etwa mit dem Schreiben als akademische Wissensproduktion selbst einhergehen. Um alternative Stimmen hörbar machen zu können, gilt es nicht zuletzt, auch die eigene Stimme (wieder) zu finden.

Aus methodologischer Sicht ist dies wenig kontrovers, wenn es darum geht, die eigene Situiertheit explizit zu machen und zu diskutieren. Wir glauben aber darüber hinaus, dass es die alltäglichen Praktiken in einer akademischen Wissensökonomie sind, die besonderer Aufmerksamkeit bedürfen, sei es bei der Formulierung von Forschungsfragen, beim wirklichen Zuhören in der Feldforschung, im Prozess des akademischen Schreibens oder in der Lehre. Aus diesem Grund verstehen wir diesen Beitrag nur als einen ersten Schritt auf dem Weg zu einer Formulierung eines Forschungsprogramms für eine kritische Normenforschung. 


\section{Zusammenfassung: Möglichkeiten einer kritischen Normenforschung}

Das hier vorgeschlagene Forschungsprogramm ist als Versuch gedacht, einen Kontrapunkt zur konstruktivistischen Normenforschung zu setzen. Während die konstruktivistische Normenforschung vornehmlich im Westen weitgehend akzeptierte Normen untersucht, diese dabei aber unhinterfragt lässt und stärkt, indem sie ihre globale Verbreitung im Sinne eines moralischen Fortschritts lokaler Gesellschaften begreift, schlagen wir vor, diese Normen konsequent zu historisieren und in ihren Geltungsansprüchen $\mathrm{zu}$ hinterfragen. Unser kritisches Forschungsprogramm zielt darauf ab, Macht- und Herrschaftsaspekte globaler Normdiffusion zu betonen und die Kontingenz von Wertbeständen aufzuzeigen. Hierzu haben wir auf poststrukturalistische, feministische, postkoloniale und autoethnographische Perspektiven und Forschungspraktiken zurückgegriffen. Mit diesen Perspektiven plädieren wir für eine kritische Sichtweise auf politische Phänomene und soziale Realität sowie eine stärkere Reflexivität sozialwissenschaftlicher Forschung. Sicherlich sind diese Forschungspraktiken nicht die einzigen, die einer kritischen Normenforschung dienlich sein können, doch bieten sie unserer Ansicht nach einen fruchtbaren Zugriff und eine kritische politische Alternative zur weitverbreiteten Praxis konstruktivistischer Forschung.

\section{Epilog}

Prof. Sapere: Das ist ja alles schön und gut, doch können Sie damit tatsächlich Sozialwissenschaften re-politisieren und wenn ja: ist dies wirklich wünschenswert? Insgesamt sind Ihre Kritik und Ihr Vorschlag vielleicht nachvollziehbar, trotzdem habe ich meine Bedenken. Auf der einen Seite würden die meisten IB-Wissenschaftler sicherlich Ihre Forderung nach mehr Reflexivität in der eigenen Forschung unterschreiben. Aber Ihre poststrukturalistische Perspektive und diese Praktiken, die Sie vorschlagen, scheinen mir dann doch sehr radikal. Mit Ihren Grundannahmen wird doch im Endeffekt jede Aussage über realweltliche Entwicklungen unmöglich. Können wir es uns da nicht gleich schenken, überhaupt noch zu forschen? (Er schaut unruhig auf seine Uhr. Die Sprechstunde ist schon lange vorbei, und dieser Termin ist nicht wie die üblichen Gespräche verlaufen. Gleich beginnt Prof. Saperes Seminar, aber Sofie scheint nicht von ihren Kritikpunkten abzubringen zu sein.) Ich hab jetzt gleich meine IB-Veranstaltung, aber wir werden das nächste Woche weiter diskutieren.

Sofie nickt und verlässt etwas desillusioniert das Büro. Prof. Sapere packt hektisch seine Unterlagen ein und ist im Begriff, den Raum zu verlassen, als er plötzlich umkehrt und die Literaturliste zu den Forschungsstrategien aufhebt, die Sofie auf dem Tisch liegen gelassen hat. Sapere überfliegt sie und überlegt sich, seine Studierenden heute zu fragen, warum sie sich entschieden haben, Politikwissenschaft zu studieren. 


\section{Literatur}

Acharya, Amitav 2004: How Ideas Spread: Whose Norms Matter? Norm Localization and Institutional Change in Asian Regionalism, in: International Organization 58: 2, 239-275.

Acharya, Amitav 2011: Dialogue and Discovery: In Search of International Relations beyond the West, in: Millennium 39: 3, 619-637.

Ackerly, Brooke A./Stern, Maria/True, Jacqui (Hrsg.) 2006: Feminist Methodologies for International Relations, Cambridge.

Ackerly, Brooke/True, Jacqui 2008: Reflexivity in Practice: Power and Ethics in Feminist Research on International Relations, in: International Studies Review 10: 4, 693-707.

Adler, Emanuel 1997: Seizing the Middle Ground: Constructivism in World Politics, in: European Journal of International Relations 3: 3, 319-363.

Adler, Emanuel/Haas, Peter M. 1992: Epistemic Communities, World Order, and the Creation of a Reflective Research Program, in: International Organization 46: 1, 367-390.

Adler-Nissen, Rebecca 2014: Stigma Management in International Relations: Transgressive Identities, Norms and Order in International Society, in: International Organization 68: 1 , i.E.

Agathangelou, Anna M./Ling, L. H. M. 2004a: Power, Borders, Security, Wealth: Lessons of Violence and Desire from September 11, in: International Studies Quarterly 48: 3, 517-538.

Agathangelou, Anna M./Ling, L. H. M. 2004b: The House of IR: From Family Power Politics to the Poisies of Worldism, in: International Studies Review 6: 4, 21-50.

Agathangelou, Anna M./Ling, L. H. M. 2005: Power and Play through Poisies: Reconstructing Self and Other in the 9/11 Commission Report, in: Millennium 33: 3, 827-853.

Agathangelou, Anna M./Ling, L. H. M. 2009: Transforming World Politics: From Empire to Multiple Worlds, London.

Albert, Mathias 1994: Postmoderne und Theorie der internationalen Beziehungen, in: Zeitschrift für Internationale Beziehungen 1: 1, 45-63.

Amy, Lori 2011: Listening for the Elsewhere and the Not-Yet: Academic Labor as a Matter of Ethical Witness, in: Inayatullah 2011a, 103-117.

Aronowitz, Stanley 1988: Science as Power: Discourse of Ideology in Modern Society, Minneapolis, MN.

Ashley, Richard K./Walker, R. B. J. 1990a: Introduction: Speaking the Language of Exile: Dissident Thought in International Studies, in: International Studies Quarterly 34: 3, 259-268.

Ashley, Richard K./Walker, R. B. J. 1990b: Conclusion: Reading Dissidence/Writing the Discipline: Crisis and the Question of Sovereignty in International Studies, in: International Studies Quarterly 34: 3, 367-416.

Barkin, J. S./Cronin, Bruce 1994: The State and the Nation: Changing Norms and the Rules of Sovereignty in International Relations, in: International Organization 48: 1, 107-130.

Bhambra, Gurminder K./Shilliam, Robbie (Hrsg.) 2009: Silencing Human Rights: Critical Engagements with a Contested Project, New York, NY.

Bleiker, Roland/Brigg, Morgan 2010: Introduction to the RIS Forum on Autoethnography and International Relations, in: Review of International Studies 36: 3, 777-778.

Brigg, Morgan/Bleiker, Roland 2010: Autoethnographic International Relations: Exploring the Self as a Source of Knowledge, in: Review of International Studies 36: 3, 778-798.

Campbell, David 1992: Writing Security: United States Foreign Policy and the Politics of Identity, Manchester.

Chakrabarty, Dipesh 1992: Postcoloniality and the Artifice of History: Who Speaks for »Indian« Pasts?, in: Representations 37, 1-26.

Chakrabarty, Dipesh 2002: Europa provinzialisieren. Postkolonialität und die Kritik der Geschichte, in: Conrad, Sebastian/Randeria, Shalini (Hrsg.): Jenseits des Eurozentrismus. 
Postkoloniale Perspektiven in den Geschichts- und Kulturwissenschaften, Frankfurt a. M., 283-312.

Chan, Stephen 2011: Accidental Scholarship and the Myth of Objectivity, in: Inayatullah 2011a, 13-18.

Checkel, Jeffrey T. 1998: The Constructivist Turn in International Relations Theory, in: World Politics 50: 2, 324-348.

Checkel, Jeffrey T. 1999: Norms, Institutions, and National Identity in Contemporary Europe, in: International Studies Quarterly 43: 1, 83-114.

Chowdhry, Geeta 2007: Edward Said and Contrapuntal Reading: Implications for Critical Interventions in International Relations, in: Millennium 36: 1, 101-116.

Christodoulidis, Emilios/Veitch, Scott 2007: Introduction, in: Veitch, Scott (Hrsg.): Law and the Politics of Reconciliation, Aldershot, 1-8.

Cohn, Carol 1987: Sex and Death in the Rational World of Defense Intellectuals, in: Signs: Journal of Women in Culture and Society 12: 4, 687-718.

Cohn, Carol 2006: Motives and Methods: Using Multi-Sited Ethnography to Study US National Security Discourses, in: Ackerly/Stern/True 2006, 91-107.

Cox, Robert W. 1981: Social Forces, States and World Orders: Beyond International Relations Theory, in: Millennium 10: 2, 126-155.

Dauphinee, Elizabeth 2010: The Ethics of Autoethnography, in: Review of International Studies 36: 3, 799-818.

Deitelhoff, Nicole 2006: Überzeugung in der Politik. Grundzüge einer Diskurstheorie internationalen Regierens, Frankfurt a. M.

Deitelhoff, Nicole 2009: The Discursive Process of Legalization: Charting Islands of Persuasion in the ICC Case, in: International Organization 63: 1, 33-65.

de Klerk, Frederik Willem 2007: Presidential Inaugural Address 1989, in: Doxtader, Erik/ Salazar, Philippe-Joseph (Hrsg.): Truth and Reconciliation in South Africa: The Fundamental Documents, Claremont, CA, 65-68.

Der Derian, James/Shapiro, Michael J. (Hrsg.) 1989: International/Intertextual Relations: Postmodern Readings of World Politics, Lexington.

Diez, Thomas 1998: Perspektivenwechsel: Warum ein »postmoderner« Ansatz für die Integrationsforschung doch relevant ist, in: Zeitschrift für Internationale Beziehungen 5: 1, 139-148.

Doty, Roxanne L. 1996: Imperial Encounters: The Politics of Representation in North-South Relations, Minneapolis, MN.

Doty, Roxanne L. 2004: Maladies of Our Souls: Identity and Voice in the Writing of Academic International Relations, in: Cambridge Review of International Affairs 17: 2, 377-392.

Doty, Roxanne L. 2006: Fronteras Compasivas and the Ethics of Unconditional Hospitality, in: Millennium 35: 1, 53-74.

Doty, Roxanne L. 2010: Autoethnography: Making Human Connections, in: Review of International Studies 36: 4, 1047-1050.

Edkins, Jenny 2011: Objects among Objects, in: Inayatullah 2011, 19-30.

El Alaoui, Khadija F. 2011: Scenes of Obscenity: The Meaning of America under Epistemic and Military Violence, in: Inayatullah 2011a, 41-55.

Engelkamp, Stephan/Glaab, Katharina 2011: Writing Norms: Norm Research as Political Practice (Millennium Annual Conference, London School of Economics, London, 22.-23.10.2011), unv. Ms.

Epstein, Charlotte 2008: The Power of Words in International Relations: Birth of an AntiWhaling Discourse, Cambridge.

Epstein, Charlotte 2012: Stop Telling Us How to Behave: Socialization or Infantilization?, in: International Studies Perspectives 13: 2, 135-145. 
Finnemore, Martha 1996: Constructing Norms of Humanitarian Intervention, in: Katzenstein, Peter J. (Hrsg.): The Culture of National Security: Norms and Identity in World Politics, New York, NY, 153-185.

Finnemore, Martha/Sikkink, Kathryn 1998: International Norm Dynamics and Political Change, in: International Organization 52: 4, 887-917.

Finnemore, Martha/Sikkink, Kathryn 2001: Taking Stock: The Constructivist Research Program in International Relations and Comparative Politics, in: Annual Review of Political Science 4, 391-416.

Florini, Ann 1996: The Evolution of International Norms, in: International Studies Quarterly 40: 3, 391-416.

Forschungsgruppe Menschenrechte 1998: Internationale Menschenrechtsnormen, transnationale Netzwerke und politischer Wandel in den Ländern des Südens, in: Zeitschrift für Internationale Beziehungen 5: 1, 5-41.

Foucault, Michel/Gordon, Colin 1980: Power/Knowledge: Selected Interviews and Other Writings: 1972-1977, New York, NY.

Gallagher, Julia 2012: Chopping the World into Bits: Africa, the World Bank and the Good Governance Norm, unv. Ms.

Gantzel, Klaus Jürgen (Hrsg.) 1975: Herrschaft und Befreiung in der Weltgesellschaft, Frankfurt a. M.

Geis, Anna 2011: Of Bright Sides and Dark Sides: Democratic Peace beyond Triumphalism, in: International Relations 25: 2, 164-170.

Glaab, Katharina/Engelkamp, Stephan 2012: New Songs for Norm Research: Gandhi, Violence and Resistance in the Politics of Agricultural Biotechnology in India, unv. Ms.

Grovogui, Siba N. 2010: Postcolonialism, in: Dunne, Timothy/Kurki, Milja/Smith, Steve (Hrsg.): International Relations Theories: Discipline and Diversity, Oxford, 238-256.

Grovogui, Siba N. 2011: To the Orphaned, Dispossessed, and Illegitimate Children: Human Rights Beyond Republican and Liberal Traditions, in: Indiana Journal of Global Legal Studies 18: 1, 41-63.

Hafner Burton, Emilie M./Tsutsui, Kiyoteru 2005: Human Rights in a Globalizing World: The Paradox of Empty Promises, in: American Journal of Sociology 110: 5, 1373-1411.

Heller, Regina/Kahl, Martin/Pisoiu, Daniela 2012: The $»$ Dark Side« of Normative Argumentation: The Case of Counterterrorism Policy, in: Global Constitutionalism 1: 2, 278-312.

Hirsch, Michal B.-J. 2007: Agents of Truth and Justice: Truth Commissions and the Transitional Justice Epistemic Community, in: Chandler, David/Heins, Volker (Hrsg.): Rethinking Ethical Foreign Policy: Pitfalls, Possibilities and Paradoxes, London, 184-205.

Holzinger, Katharina/Knill, Christoph/Jörgens, Helge (Hrsg.) 2007: Transfer, Diffusion und Konvergenz von Politiken (PVS-Sonderheft), Wiesbaden.

Howarth, David/Stavrakakis, Yannis 2000: Introducing Discourse Theory and Political Analysis, in: Howarth, David/Norval, Aletta/Stavrakakis, Yannis (Hrsg.): Discourse Theory and Political Analysis: Identities, Hegemonies and Social Change, Manchester, 1-23.

Hülsse, Rainer 2011: I, the Double Soldier: An Autobiographic Case-Study on the Pitfalls of Dual Citizenship, in: Inayatullah 2011a, 56-64.

Humphrey, Michael 2002: The Politics of Atrocity and Reconciliation: From Terror to Trauma, New York, NY.

Inayatullah, Naeem/Blaney, David L. 2004: International Relations and the Problem of Difference, New York, NY.

Inayatullah, Naeem (Hrsg.) 2011a: Autobiographical International Relations: I, IR, New York, NY.

Inayatullah, Naeem 2011b: Falling and Flying: An Introduction, in: Inayatullah 2011a, 1-12.

Inayatullah, Naeem/Blaney, David L. 2012a: The Dark Heart of Kindness: The Social Construction of Deflection, in: International Studies Perspectives 13: 2, 164-175. 
Inayatullah, Naeem/Blaney, David L. 2012b: Liberal Fundamentals: Invisible, Invasive, Artful, and Bloody Hands, in: Journal of International Relations and Development 15: 2, 290-315.

Jackson, Patrick T. 2011: Three Stories: A Way of Being in the World, in: Inayatullah 2011a, 161-172.

Kairos 2007: The Kairos Document, in: Doxtader, Erik/Salazar Philippe-Joseph (Hrsg.): Truth and Reconciliation in South Africa: The Fundamental Documents, Claremont, CA, 50-56.

Katzenstein, Peter J. 1996: Introduction: Alternative Perspectives on National Security, in: Katzenstein, Peter J. (Hrsg.): The Culture of National Security: Norms and Identity in World Politics, New York, NY, 1-32.

Keck, Margaret W./Sikkink, Kathryn 1998: Activists Beyond Borders: Advocacy Networks in International Politics, London.

Keohane, Robert O. 1988: International Institutions: Two Approaches, in: International Studies Quarterly 32: 4, 379-396.

Klotz, Audie 1995: Norms in International Relations: The Struggle Against Apartheid, London.

Kowert, Paul/Legro, Jeffrey 1996: Norms, Identity, and Their Limits: A Theoretical Reprise, in: Katzenstein, Peter J. (Hrsg.): The Culture of National Security: Norms and Identity in World Politics, New York, NY, 451-487.

Kronsell, Annica 2006: Methods for Studying Silences: Gender Analysis in Institutions of Hegemonic Masculinity, in: Ackerly/Stern/True 2006, 108-128.

Krook, Mona L./True, Jacqui 2012: Rethinking the Life Cycles of International Norms: The United Nations and the Global Promotion of Gender Equality, in: European Journal of International Relations 18: 1, 103-127.

Kruzel, Joseph/Rosenau, James N. (Hrsg.) 1989: Journeys through World Politics: Autobiographical Reflections of Thirty-Four Academic Travelers, Lexington.

Kumarakulasingam, Narendran 2011: Stammers between Silence and Speech, in: Inayatullah 2011a, 31-40.

Laclau, Ernesto/Mouffe, Chantal 2001: Hegemony and Socialist Strategy: Towards a Radical Democratic Politics, London.

Litfin, Karen T. 1994: Ozone Discourses: Science and Politics in Global Environmental Cooperation, New York, NY.

Löwenheim, Oded 2010: The »I« in IR: An Autoethnographic Account, in: Review of International Studies 36: 4, 1023-1045.

MacKenzie, Megan/Sesay, Mohamed 2012: No Amnesty from/for the International: The Production and Promotion of TRCs as an International Norm in Sierra Leone, in: International Studies Perspectives 13: 2, 146-163.

March, James G./Olsen, Johan P. 1998: The Institutional Dynamics of International Political Orders, in: International Organization 52: 4, 943-969.

Moon, Claire 2008: Narrating Political Reconciliation: South Africa's Truth and Reconciliation Commission, Plymouth.

Nandy, Ashis 1988: The Intimate Enemy: Loss and Recovery of Self under Colonialism, New Delhi.

Neumann, Iver B./Sending, Ole Jacob 2010: Governing the Global Polity: Practice, Mentality, Rationality, Ann Arbor, MI.

Panke, Diana/Petersohn, Ulrich 2011: Why International Norms Disappear Sometimes, in: European Journal of International Relations, doi:10.1177/1354066111407690.

Park, Susan 2005: Norm Diffusion within International Organizations: A Case Study of the World Bank, in: Journal of International Relations and Development 8: 2, 111-141.

Park-Kang, Sungju 2011: Utmost Listening: Feminist IR as a Foreign Language, in: Millennium 39: 3, 861-877. 
Payne, Roger A. 2001: Persuasion, Frames and Norm Construction, in: European Journal of International Relations 7: 1, 37-61.

Peterson, V. Spike 1990: Whose Rights? A Critique of the »Givens« in Human Rights Discourse, in: Alternatives 15: 3, 303-344.

Platenkamp, Josephus D. 2007: Cultural Values, Human Rights and Peacekeeping Tasks: Some Anthropological Considerations, in: Militaire Spectator 176: 3, 96-104.

Randeria, Shalini 2002: Entangled Histories of Uneven Modernities: Civil Society, Caste Solidarities and Legal Pluralism in Post-Colonial India, in: Elkana, Yehuda/Krastev, Ivan/ Macamo, Elisio/Randeria, Shalini (Hrsg.): Unraveling Ties: From Social Cohesion to New Practices of Connectedness, New York, NY.

Renner, Judith 2013: Discourse, Normative Change, and the Quest for Reconciliation in Global Politics, Manchester, i.E.

Reus-Smit, Christian 2001: Human Rights and the Social Construction of Sovereignty, in: Review of International Studies 27: 4, 519-538.

Risse, Thomas 2003: Konstruktivismus, Rationalismus und Theorien Internationaler Beziehungen - warum empirisch nichts so heiß gegessen wird, wie es theoretisch gekocht wurde, in: Hellmann, Gunther/Wolf, Klaus D./Zürn, Michael (Hrsg.): Die neuen internationalen Beziehungen. Forschungsstand und Perspektiven in Deutschland, Baden-Baden, 99-132.

Risse, Thomas 2006: Transnational Actors and World Politics, in: Carlsnaes, Walter/Risse, Thomas/Simmons, Beth A. (Hrsg.): Handbook of International Relations, London, 255-274.

Risse, Thomas/Sikkink, Kathryn 1999: The Socialization of International Human Rights Norms into Domestic Practices: Introduction, in: Risse/Ropp/Sikkink 1999, 1-38.

Risse, Thomas/Ropp, Stephen C./Sikkink, Kathryn (Hrsg.) 1999: The Power of Human Rights: International Norms and Domestic Change, Cambridge.

Said, Edward W. 1994: Kultur und Imperialismus. Einbildungskraft und Politik im Zeitalter der Macht, Frankfurt a. M.

Sandholtz, Wayne 2008: Dynamics of International Norm Change: Rules against Wartime Plunder, in: European Journal of International Relations 14: 1, 101-131.

Sandholtz, Wayne/Stiles, Kendall W. 2009: International Norms and Cycles of Change, Oxford.

Schlichte, Klaus/Wilke, Boris 2000: Der Staat und einige seiner Zeitgenossen: Zur Zukunft des Regierens in der »Dritten Welt«, in: Zeitschrift für Internationale Beziehungen 7: 2, 359-384.

Shaw, Rosalind 2005: Rethinking Truth and Reconciliation Commissions: Lessons from Sierra Leone (United States Institute of Peace Special Report 130), in: http://www.usip.org/ files/resources/sr130.pdf; 24.09.2012.

Smith, Steve 2004: Singing Our World into Existence: International Relations Theory and September 11, in: International Studies Quarterly 48: 3, 499-515.

Smith, Tony 2011: Democratic Peace Theory: From Promising Theory to Dangerous Practice, in: International Relations 25: 2, 151-157.

Spry, Tami 2011: Performing Autoethnography: An Embodied Methodological Praxis, in: Qualitative Inquiry 7: 6, 706-732.

Stump, Jacob L. 2011: Weakness Leaving My Body: An Essay on the Interpersonal Relations of International Politics, in: Inayatullah 2011a, 65-77.

Sylvester, Christine 2000: Feminist Theory and International Relations in a Postmodern Era, Cambridge.

Tickner, J. Ann 1997: You Just Don't Understand: Troubled Engagements between Feminists and IR Theorists, in: International Studies Quarterly 41: 4, 611-632.

Torfing, Jacob 2005: Discourse Theory: Achievements, Arguments, and Challenges, in: Howarth, David/Torfing, Jacob (Hrsg.): Discourse Theory in European Politics: Identities, Policy and Governance, Hampshire, 1-33. 
Towns, Ann E. 2010: Women and States. Norms and Hierarchies in International Society, Cambridge.

Ulbert, Cornelia 1997: Ideen, Institutionen und Kultur. Die Konstruktion (inter-)nationaler Klimapolitik in der BRD und in den USA, in: Zeitschrift für Internationale Beziehungen 4: 1, 9-40.

van Kersbergen, Kees/Verbeek, Bertjan 2007: The Politics of International Norms: Subsidiarity and the Imperfect Competence Regime of the European Union, in: European Journal of International Relations 13: 2, 217-238.

Walker, R. B. J. 1993: Inside/Outside: International Relations as Political Theory, Cambridge.

Widmaier, Wesley W./Park, Susan 2012: Differences Beyond Theory: Structural, Strategic, and Sentimental Approaches to Normative Change, in: International Studies Perspectives 13: 2, 123-134.

Wiener, Antje 2007: The Dual Quality of Norms and Governance beyond the State: Sociological and Normative Approaches to »Interaction«, in: Critical Review of International Social and Political Philosophy 10: 1, 47-69.

Wiener, Antje 2009: Enacting Meaning-In-Use: Qualitative Research on Norms and International Relations, in: Review of International Studies 35: 1, 175-193.

Wiener, Antje 2010: Zur normativen Wende in den IB. Triangulation of a Different Kind, in: Zeitschrift für Internationale Beziehungen 17: 2, 335-354.

Zehfuss, Maja 1998: Sprachlosigkeit schränkt ein: Zur Bedeutung von Sprache in konstruktivistischen Theorien, in: Zeitschrift für Internationale Beziehungen 5: 1, 109-137.

Zehfuss, Maja 2002: Constructivism in International Relations: The Politics of Reality, Cambridge.

Zimmermann, Lisbeth 2012: Global Norms with a Local Face? The Interaction of Rule of Law and Norm Translation in Guatemala (Dissertationsschrift), Darmstadt. 